\title{
Otitis Media with ANCA-associated Vasculitis: A New Concept and the Associated Criteria
}

\author{
Yusuke Kobari and Tasuku Nagasawa
}

\begin{abstract}
:
A previously healthy 77-year-old Japanese man presented with a 2-week history of daily fevers peaking at $38^{\circ} \mathrm{C}$, chills, hearing loss, and almost $10 \mathrm{~kg}$ of unintentional weight loss over 2 months. Pure tone audiometry showed mixed conductive-sensorineural hearing loss: right, $63.6 \mathrm{~dB}$, left, $80.0 \mathrm{~dB}$. Blood tests after admission showed a high myeloperoxidase-antineutrophil cytoplasmic antibody (MPO-ANCA) level ( $>300 \mathrm{U} /$ $\mathrm{mL}$ ), so we suspected ANCA-related vasculitis. The Japanese Otorhinolaryngology Society has recently been advocating the concept of otitis media with ANCA-associated vasculitis (OMAAV). Our case met the criteria proposed, leading to our diagnosis.
\end{abstract}

Key words: ANCA-related vasculitis, MPO-ANCA, OMAAV

(Intern Med 56: 3365-3367, 2017)

(DOI: 10.2169/internalmedicine.8704-16)

\section{Introduction}

The symptoms of antineutrophil cytoplasmic antibody (ANCA)-related vasculitis (AAV) varies reflected by the systemic vasculitis. Hearing loss as the initial symptom of AAV is rare, but the prognosis of hearing ability is not so good. As the hearing loss may lead to deafness, and the hearing ability cannot be recovered, it is very important to consider AAV as the differencial diagnosis and start treatment as early as possible, if we see the patient with hearing loss.We describe the case of a patient presented with mixed conductive-sensorineural hearing loss with positive MPOANCA that illustrates the new aspects of ANCA-related vasculitis.

\section{Case Report}

In October, 2013, a previously healthy 77-year-old Japanese man presented with a 2-week history of daily fevers peaking at $38^{\circ} \mathrm{C}$, chills, hearing loss, anorexia, loss of motivation, and almost $10 \mathrm{~kg}$ of unintentional weight loss over 2 months. He had begun wearing a hearing aid three days before admission. He had no remarkable medical history or prescriptions. On an examination, he was alert and con- scious. Jolt accentuation was negative, and he did not have a stiff neck. The finger-nose test result was slightly poor for both hands. He could not walk with a tandem gait. He had hearing impairment in both ears but showed no muscle weakness. The findings on other examinations were normal.

The complete blood count and white-cell differential count were normal, as were the blood levels of electrolytes and glucose. The results of tests of the renal and liver function were also normal. He had elevated C-reactive protein levels $(16.3 \mathrm{mg} / \mathrm{dL})$, but other blood test results, including ferritin, KL-6, $\beta$-D glucan, and tumor markers (CEA and CA 19-9) and serology for hepatitis B, hepatitis C, and HIV, were normal. Pure tone audiometry showed mixed conductive-sensorineural hearing loss: right, $63.6 \mathrm{~dB}$ (bone conduction: $36.3 \mathrm{~dB}$ ), left, $80.0 \mathrm{~dB}$ (bone conduction: 52.5 dB) (Fig. 1, Left). The hypertrophy of the tympanum and exudates fluid in the middle ear were found by an otoscope. A biopsy of the middle ear was not performed. Ear computed tomography (CT) showed soft tissue in both middle ear cavities and the tympanum as well as mastoid cells (Fig. 2), indicating exudative otitis media. There was no destruction or disconnection of ossicles. Because of pyrexia of unknown origin, we cultured blood, urine, and cerebrospinal fluid samples, which were negative. Mycobacterium cultures of sputum and gastric secretions and the QuantiFERON ${ }^{\circledR}$-TB 

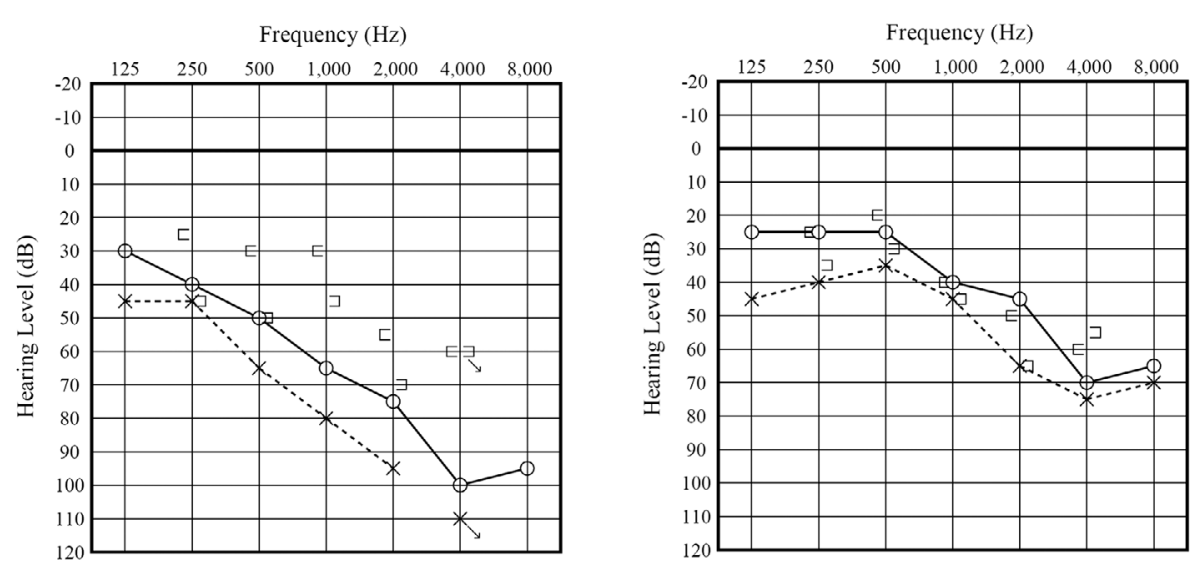

Figure 1. Auditory capacity. At admission (on the left). Right: $63.6 \mathrm{~dB}$ (bone conduction: $36.3 \mathrm{~dB}$ ), left: $80.0 \mathrm{~dB}$ (bone conduction: $52.5 \mathrm{~dB}$ ). When discharged (on the right). The auditory capacity improved to right $37.5 \mathrm{~dB}$ and left $47.5 \mathrm{~dB}$. $O$ : right air conduction, $\square$ : right bone conduction, $\times:$ left air conduction, $\sqsupset$ : left bone conduction.

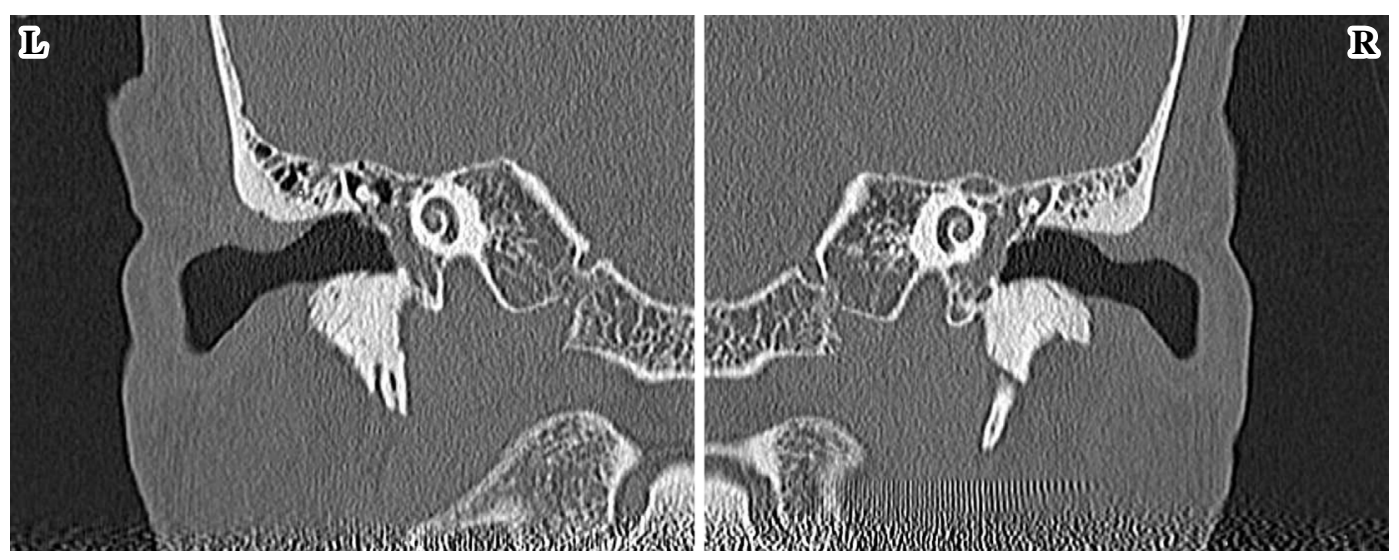

Figure 2. Ear CT showing soft tissue in both middle ear cavities and the tympanum as well as mastoid cells.

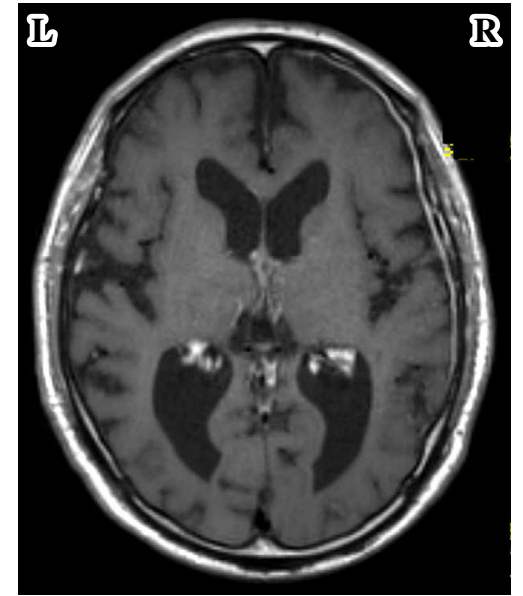

Figure 3. Contrast-enhanced magnetic resonance imaging (MRI) of the head was normal. There was no evidence of hypertrophic pachymeningitis, fresh infarction, or mass lesions. An aneurysm of the right internal carotid artery was suspected (image not shown), but no further testing, such as angiography or CT scans, was performed.
Gold (Cellestis, Carnegie, Australia) test were also negative. No vegetations were found on endothoracic echocardiography. Blood KL-6, SP-A, and SP-D levels were within normal limits, and an X-ray examination showed that interstitial pneumonia was unlikely. There were no mass lesions observed on CT. Blood tests after admission showed a high myeloperoxidase-antineutrophil cytoplasmic antibody (MPOANCA) level (>300 U/mL), so we suspected ANCA-related vasculitis. There was no hypertrophic pachymeningitis on contrast-enhanced magnetic resonance imaging (MRI) (Fig. 3) or eye lesions.

Fourteen days after admission, we started prednisolone (PSL) at $40 \mathrm{mg} /$ day and observed marked clinical improvement, a decreased fever, and the disappearance of cough the next day. The finger-nose test result returned to normal, and he was able to walk with a tandem gait. The auditory capacity gradually improved, so we reduced the PSL dosage to 20 $\mathrm{mg}$ /day and added azathioprine $50 \mathrm{mg} /$ day. The auditory capacity improved to $37.5 \mathrm{~dB}$ (right) and $47.5 \mathrm{~dB}$ (left) (Fig. 1, Right). No conductive-sensorineural mismatch was found. We therefore diagnosed the patient with ANCArelated vasculitis with chief complaints of pyrexia and hear- 
Table. Criteria of Otitis Media ANCA-associated Vasculitis (proposal).

1. Otitis media refractory to antibiotics or tympanostomy tube

2. Progression of the bone conduction threshold

3. Serum PR3-ANCA or MPO-ANCA is positive

4. (1) or (2) is seen by a biopsy

(1) Necrotizing granulomatous inflammation with giant cells

(2) Necrotizing granulomatous vasculitis in the small or middle arteries

5. The diseases below are negative

(1) Otitis media with tuberculosis

(2) Cholesterin granuloma

(3) Eosinophilic otitis media

(4) Tumor

6. Reference complications or secondary illnesses

(1) Upper respiratory (without ear), lung, or kidney lesion

(2) Facial nerve palsy

(3) Hypertrophic pachymeningitis

(4) Multiple mononeuropathy

(5) Subarachnoid hemorrhage

Definite: $(1$ or 2$)+(3$ or 4$)$

Likely: $1+2+5$ and prednisolone, cyclophosphamide, or others are effective

ANCA: anti neutrophil cytoplasmic antibody

ing loss.

\section{Discussion}

Diseases with sensorineural hearing loss include acute/ chronic otitis media, cholesteatoma, meningitis, otitis interna (by viral infection, meningitis, or otitis media), Meniere's disease, sudden sensorineural hearing loss, drugs, otosclerosis, acoustic neuroma, trauma and perilymph fistula. In this case, otoscopy showed bilateral thickening of the tympanic membrane and pooling of the exudate, suggestive of serous otitis media. However, because of his sustained fever and weight loss, we suspected a tumor or auto-immune disease. MPO-ANCA was positive, so we diagnosed the patient with ANCA-related vasculitis. Except for the MPO-ANCApositive finding, the patient met the criteria for limited granulomatosis with polyangiitis (GPA). Watts' criteria also supported this diagnosis (1). It has been reported that GPA with serous otitis media and sensorineural hearing loss is accompanied by more complications, such as palsy of facial nerve or hypertrophic pachymeningitis, than GPA without serous otitis media (2).

Initial hearing loss is reported in 47\% ANCA-related vasculitis patients, a value much higher than expected (3). However, another report of 32 ANCA-related vasculitis patients with refractory otitis media stated that only 14 (44\%) met the criteria of ANCA-related vasculitis established by the Japanese Ministry of Health, Labour and Welfare group and that it was hard to make a diagnosis in other patients because MPO-ANCA was not mentioned, leading to the late initiation of treatment. For this reason, the Japanese Otorhinolaryngology Society has been advocating the concept of otitis media with ANCA-associated vasculitis (OMAAV). The OMMAV criteria are shown in Table (4). In our case, Criteria 1, 2, 3, and 5 were present. However, whether or not OMAAV is an independent disease of AAV remains controversial. Indeed, in many cases of OMAAV, granuloma exists in the middle ear, and as it grows, it destroys ossicles.

This implies that OMAAV is only a complication of GPA and that OMAAV may therefore be diagnosed as being a subtype of GPA.

Further accumulation of such cases is therefore required.

Considering the standard treatment for GPA, we selected PSL and azathioprine to treat the present case. The EULAR recommends the use of MTX or MMF as first-line drugs for AAV, but in Japan, the use of MTX for AAV is not covered by insurance. Fortunately, we were able to control the activity of AAV using PSL, but the prolonged use of PSL causes many drug-related complications, so we added azathioprine in order to reduce the dosage of PSL.

A reduced hearing ability tends to either persist or worsen, not recover, and a delayed diagnosis can lead to severe complications, like deafness, palsy of facial nerves, or hypertrophic pachymeningitis. In our case, there were no lung or kidney lesions. However, this case was atypical in that the chief complaint was hearing loss and a fever. Hearing loss may lead to deafness, and the hearing ability cannot be recovered, so as in our case, it is very important to diagnose ANCA-associated vasculitis and start treatment as early as possible.

Informed consent was obtained from the patient.

The authors state that they have no Conflict of Interest (COI).

\section{References}

1. Watts R, Lane S, Hanslik T, et al. Development and validation of a consensus methodology for the classification of the ANCAassociated vasculitides and polyarteritis nodosa for epidemiological studies. Ann Rheum Dis 66: 222-227, 2007.

2. Osaki S, Andou T, Iseki K, et al. Guideline for management of vasculitis syndrome (JCS 2008). Circ J 75: 474-503, 2008.

3. Harabuchi Y, Kishibe K, Komabayashi Y. Clinical manifestations of granulomatosis with polyangiitis (GPA: Wegener's granulomatosis) in the upper respiratory tracts by otolaryngologists of Japan. Clin Exp Nephrol 17: 663-666, 2013.

4. Harabuchi Y, Kishibe K. Otitis media with ANCA-associated vasculitis. Practica Oto-Rhino-Laryngologica 107: 587-598, 2014.

The Internal Medicine is an Open Access article distributed under the Creative Commons Attribution-NonCommercial-NoDerivatives 4.0 International License. To view the details of this license, please visit (https://creativecommons.org/licenses/ by-nc-nd/4.0/).

(C) 2017 The Japanese Society of Internal Medicine Intern Med 56: 3365-3367, 2017 